\title{
Structural Performance Evaluation for Vibration-based Energy Harvester utilized in Railway Vehicle
}

\author{
Ki-Weon Kang ${ }^{1, a}$ and Ji-Won $\mathrm{Jin}^{2}$ \\ ${ }^{1}$ Department of Mechanical Engineering, Kunsan National University, Kunsan, Jeonbuk, Republic of Korea \\ ${ }^{2} R \& D$ Group, Jeonbuk Institute of Automotive Convergence Technology, Kunsan, Jeonbuk, Republic of Korea
}

\begin{abstract}
This study aims to assess the structural performance and structural integrity of vibration energy harvester (VEH). For this, the structural performance test were conducted to identify the natural frequency and structural response against frequency. And then, static structural analysis was performed using finite element analysis to investigate the failure critical locations (FCLs). Finally, we conducted the frequency response analysis in frequency domain to obtain the structural response with frequency and investigate the structural integrity of VEH. Using the above results, we assessed the structural performance and structural integrity of two types of VEHs.
\end{abstract}

\section{Introduction}

The vibration-based energy harvester (VEH) [1-3] uses vibrations generated from various structures or components, and in particular, maximizes the power generation using the resonance between the excitation frequency of the vibration load, which comes from structures or components, and the natural frequency of the VEH itself, thereby enhancing the efficiency of power production [4]. And the VEH is subjected to very high levels of acceleration and load because of the aforementioned resonance when the same level of vibration is applied. Hence, the VEH is very likely to be damaged structurally. It is, therefore, essential to assess the evaluation of the structural performance and integrity.

We assessed the structural performance and integrity of the VEH as shown in Fig. 1, which is utilized in high speed railway vehicle [5]. For this, a static performance test was conducted using the incremental sinusoidal sweep condition. Next, we conducted a static structural analysis to identify the natural frequency and failure critical location (FCL). We then conducted a frequency response analysis to identify the stress at the FCL against the frequency.

\section{Results and Discussion}

We investigated the structural performance and integrity for two types of VEHs (Type I and Type II). The VEH normally comprises a shaft carrying a vibration load, a coil case, a main body, a neodymium magnet and leaf-spring-shaped moving component [3]. In Type I VEH, the material of the spring was

\footnotetext{
${ }^{\mathrm{a}}$ Corresponding author : kwkang68@kunsan.ac.kr
} 
STS 410, and the thickness was $0.65 \mathrm{~mm}$. This VEH was designed to have a natural frequency of $f_{\mathrm{n}, 1}$ $\mathrm{Hz}$ with the displacement limit of $4 \mathrm{~mm}$. Type II VEH had a rubber stopper that could restrict the displacement of $2 \mathrm{~mm}$ at both ends of the spring. The material of the spring was SKD61 with thickness of $0.7 \mathrm{~mm}$ and the natural frequency of $f_{\mathrm{n}, 2} \mathrm{~Hz}$.

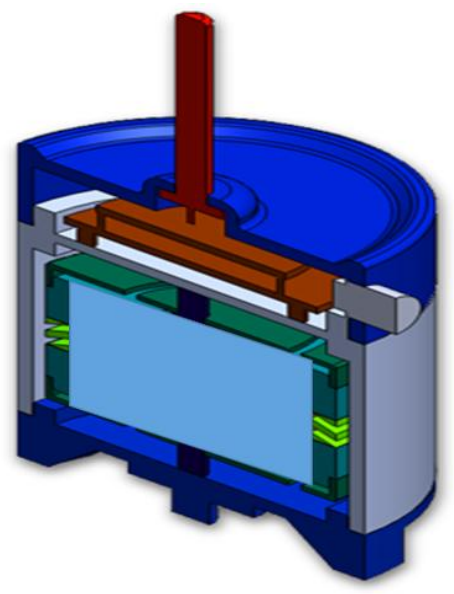

Figure 1. Vibration-based energy harvester utilized in railway vehcile

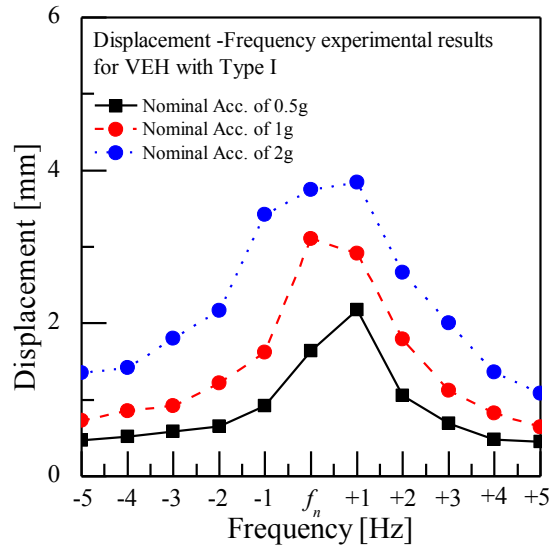

(a) Type I

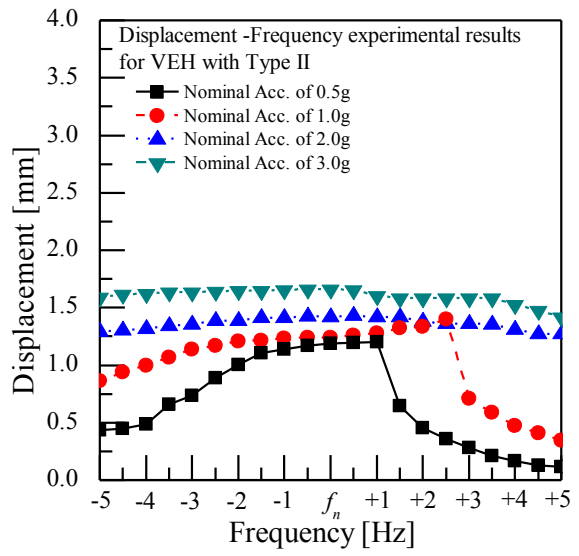

(b) Type II

Figure 2. Measured displacement-frequency curve

\subsection{Static performance test}

The static performance of the VEH was evaluated based on the incremental sinusoidal sweep tests, which their frequency ranges are natural frequencies of VEHs $\pm 5 \mathrm{~Hz}$ with an increment of $1 \mathrm{~Hz}$ and $0.5 \mathrm{~Hz}$ for Type I and II VEHs, respectively, and their accelerations levels are $0.5,1,2$, and $3 \mathrm{~g}$, respectively. Detail explanation for test procedure is in reference [6]. Fig. 2 shows the maximum displacement measured at outer rim of leaf-spring shaped moving component using the laser displacement sensor against the frequency of excitation. The first VEH shows quite different behaviour from the Type II VEH; this is attributed to that the displacement limitation of Type II VEH is just $2 \mathrm{~mm}$ but $4 \mathrm{~mm}$ for Type I VEH. 


\subsection{Static analysis}

We conducted the static analysis using FE model in Fig. 3 [5]. The finite-element model was constructed using solid (CTETRA) and shell (CTRIA3) elements; the number of elements and nodes were 49,064 and 14,507, respectively. And, the multi-point constraints (MPC) were used between the solid and shell elements to avoid the hinge connections that could result from a mismatch in degrees of freedom of the solid and shell elements.

Fig. 4 shows the stress distribution obtained from the static FE analysis. The maximum stresses occur at the outer rim of the moving component for both VEHs and their values are $31 \mathrm{MPa}$ and $16 \mathrm{MPa}$ for Type I and Type II VEHs, respectively. These stresses are quite much lower than the experimental results and this behaviour comes from that the static analysis could not take into resonance between the excitation and natural frequency of VEHs.

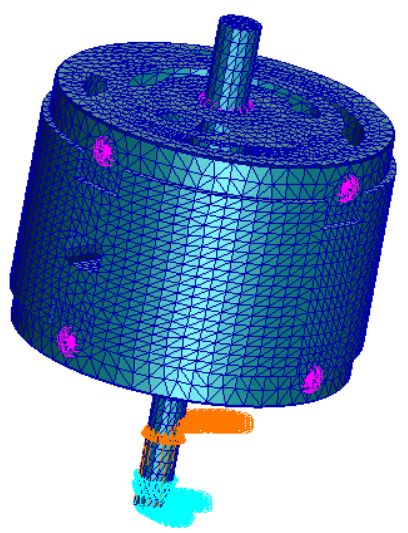

Figure 3. FE model for VEH

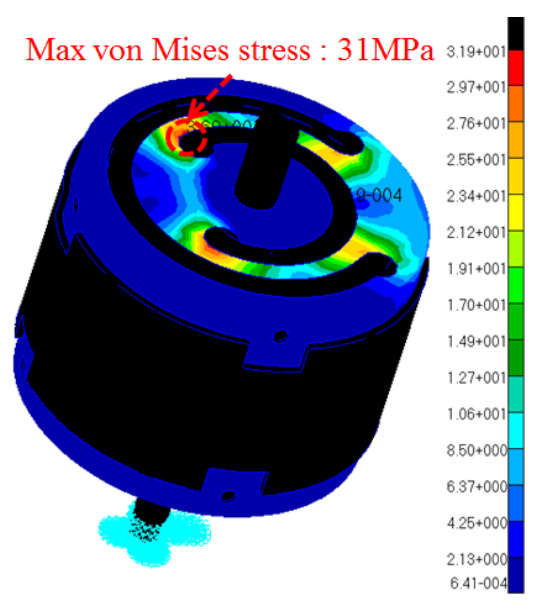

(a) Type I

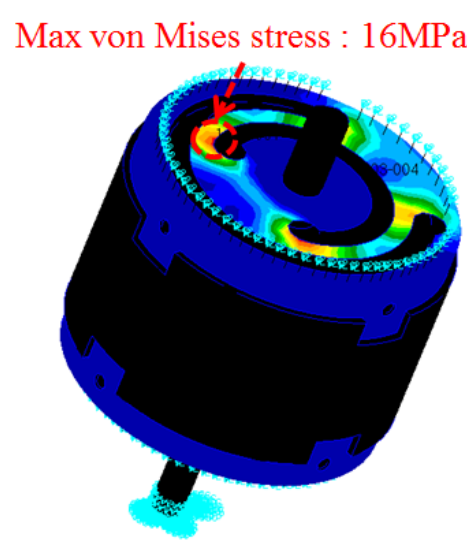

(b) Type II

Figure 4. Stress contour of VEH through static FE analysis

\subsection{Frequency response analysis}

The performance and structural integrity of vibration-based energy harvesters (VEHs) should be investigated considering the resonance between the excitation forces and natural frequency. The 
excitation forces are defined in the frequency domain and the structural response considering the resonance is expressed as the frequency response. Through the frequency response analysis, we could obtain the stress, displacement or acceleration response considering the resonance at specific locations in frequency domain.

Fig. 5 exhibits the stress contour on the leaf-spring shape moving component for Type I and Type II VEHs, respectively under the acceleration of $0.5 \mathrm{~g}$. The stress may quite differ from the static FE results in Fig. 4 and this behaviour may come from the resonance between the excitation and natural frequency in frequency response analysis. For further understanding these behaviours, the displacement - frequency response from the analysis are compared with the experimental results at the same points measured in the static performance test in Fig. 6 [5]. The figure states that the displacement-frequency results agree well with the experimental results for both the VEHs. It can be, therefore, seen that the frequency response analysis could well describe the response of the VEH with frequency.

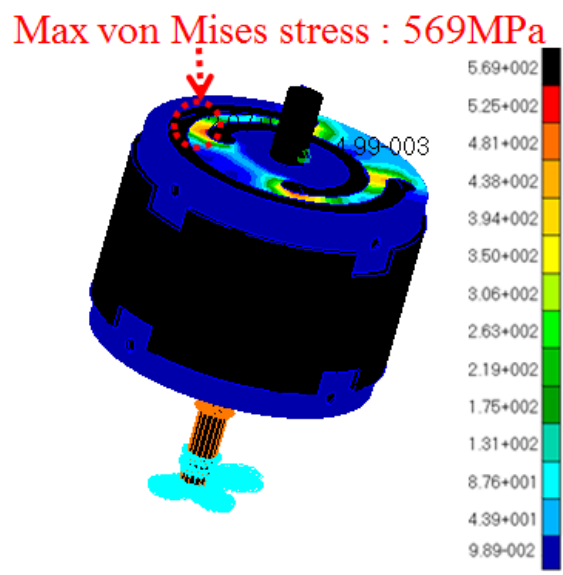

(a) Type I

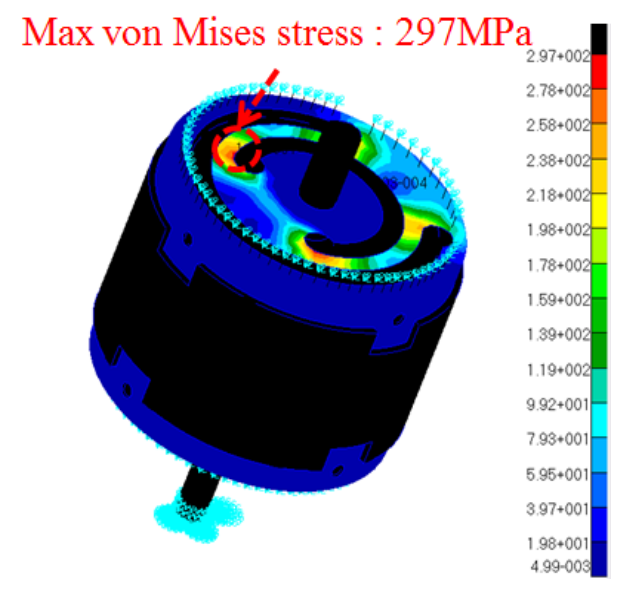

(b) Type II

Figure 5. Stress contour from frequency response analysis for VEH

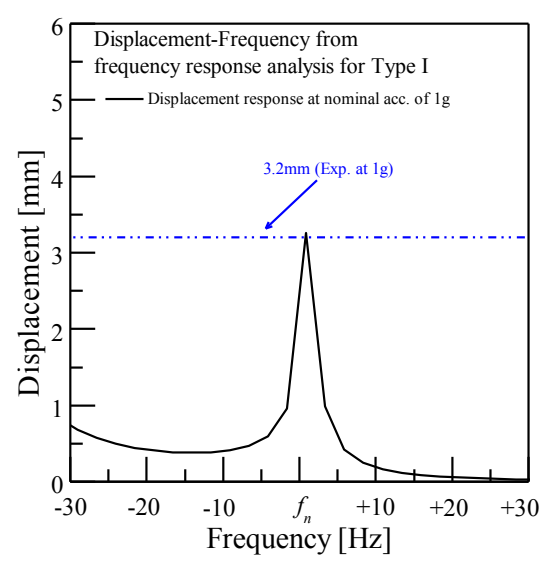

(a)Type I

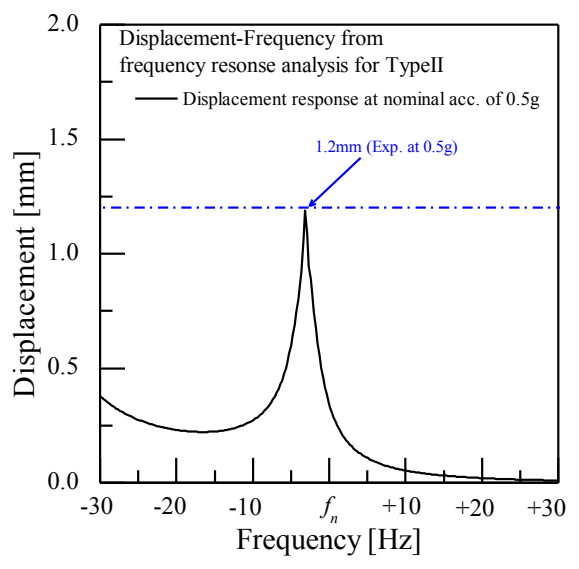

(b) Type II

Figure 6. Displacement response from frequency response analysis for VEH

\section{Conclusions}


We assessed the static performance and structural integrity of vibration-based energy harvesters. For this, we performed the static performance test, static structural analysis and frequency response analysis for two types of VEHs.

\section{Acknowledgment}

This research was supported by the Korea Institute of Energy Technology Evaluation and Planning (KETEP) and the Ministry of Trade, Industry \& Energy (MOTIE) of the Republic of Korea(No.20174030201670) and by Basic Science Research Program through the National Research

Foundation of Korea(NRF) funded by the Ministry of Education(NRF-2016R1D1A1A09918310).

\section{References}

1. S. Roundy, J. of Intelligent Material Systems and Structures, 16 (2005)

2. J.E. Kim, H. Kim, H. Yoon, Y.Y. Kim, B.D. Youn, Int'l J of Precision Engineering and Manufacturing-Green Technology, 2, (2015)

3. R. Andosca, T.G. McDonald, V. Genova, S. Rosenberg, J. Keating, C. Benedixen, J. Wu., Sensors and Actuators A: Physical, 178 (2012)

4. G. Frank, W. Peter, J. of Micromechanics and Microengineering, 18 (2008)

5. J.H. Kim, J.W. Jin, J.H. Lee, K.W. Kang, Engineering Failure Analysis, 73 (2017)

6. J.W. Jin, K.W. Kang, J.H. Kim, Int'l J. of Precision Engineering and Manufacturing - Green Technology, 2 (2015) 